\title{
Indocyanine Green-Loaded Hollow Mesoporous Silica Nanoparticles as a Novel Theranostics
}

\author{
Suk Ho Hong, Yongdoo Choi \\ Molecular Imaging and Therapy Branch, National Cancer Center \\ 323 Ilsan-ro, Goyang-si, Gyeonggi-do, Korea 10408 \\ shh@ncc.re.kr; ydchoi@ncc.re.kr
}

\section{Extended Abstract}

Indocyanine green (ICG) is a FDA-approved near-infrared (NIR) fluorophore that is widely used in in vivo nearinfrared (NIR) fluorescence imaging of cancers [1]. However, since ICG is "always on" agent, it has the potential problem of high background fluorescence when circulating in the blood stream, which can result in a low target-to-background ratio (TBR) in in vivo imaging. In addition, non-specific uptake by normal cells and tissues of ICG further reduced TBR. Due to these reasons, its applications for fluorescence imaging as well as phototherapy of cancers have been limited.

Here we developed ICG-loaded hollow mesoporous silica nanoparticles (HMSNP) for selective NIR fluorescence imaging and phototherapy of cancers. We expected that NIR fluorescence and phototoxicity are turned off in normal cells or in the extracelluar regions but becomes highly fluorescent and phototoxic inside cancer cells, thereby enabling selective fluorescence imaging and therapy of cancers. The size of the prepared nanoparticles was about $180 \mathrm{~nm}$ in diameter. ICG was loaded into HMSNP by $12 \mathrm{w} / \mathrm{w} \%$. Comparison of the UV/Vis absorption spectra of ICG-loaded HMSNP and free ICG in aqueous solution confirmed aggregation of the loaded ICGs inside the nanoparticles. As expected, both NIR fluorescence and singlet oxygen generation of ICG-loaded HMSNP were significantly inhibited in comparison with free, ICG. In the in vitro cell study, strong fluorescence was observed in the cancer cells treated with ICG-loaded HMSNP indicating turn-on of its fluorescence inside cancer cells. Upon light irradiation, ICG-loaded HMSNP showed significant cytotoxicity higher than that of free ICG molecules. These features make HMSNP a promising platform for utilizing ICG in biomedical applications.

\section{References}

[1] Y.-G. Wang et al., "Indocyanine green-loaded perfluorocarbon nanoemulsions for bimodal ${ }^{19}$ F-magnetic resonance/nearinfrared fluorescence imaging and subsequent phototherapy," Quant. Imaging Med. Surg., vol. 3, no. 3, pp. 132-140, 2013. 